\title{
О ЗАДАЧЕ ПОГРУЖЕНИЯ С ПРОИЗВОЛЬНЫМ ЯДРОМ ПОРЯДКА $p^{n}$
}

\author{
(Представил А. Хумал)
}

Пусть $F=\Gamma\left(k / k_{0}\right)-$ группа Галуа расширения полей $k / k_{0}, \quad G \stackrel{\varphi}{\rightarrow} F-$ эпиморфизм группы $G$ на группу $F$ с ядром $N$ и $\operatorname{char} k$ не делит $\operatorname{ord} N$.

Пусть $p$ - произвольное простое число и $G-p$-группа, a ord $N=p^{n}$, при этом безразлично, абелева группа $N$ или неабелева.

Будем исследовать задачу погружения, связанную-с точной последовательностью

$$
1 \rightarrow N \rightarrow G \stackrel{\varphi}{\rightarrow} F \rightarrow 1
$$

Необходимое для разрешимости этой задачи усліовие согласн̆ости найдено Д. К. Фаддеевым $\left.{ }^{1}\right]$ и переоткрыто Х. Хассе $\left[{ }^{2}\right]$. Приведем две его формулировки.

1. В групповой алгебре $k N$ существует система элементов («система согласности») $\left\{l_{\sigma}\right\}_{\sigma \in G}$ таких, что $l_{\sigma_{1}}^{\sigma_{2}} l_{\sigma_{2}}=l_{\sigma_{1} \sigma_{2}}$ и $\cdot l_{\tau}=\tau^{-1}$ при $\tau \in N$. Здесь $\sigma$ действует на элементы поля $k$ как $\varphi(\sigma)$, а на элементы $\tau \in N$ по формуле $\tau^{\sigma}=\sigma^{-1} \tau \sigma$.

2. Скрещенное произведение $A$ группы $G$ с полем $k$ есть алгебра матриц порядка ord $F$ над некоторой своей подалгеброй.

На основе этих формулировок условия согласности в статье исследуется второе препятствие для задачи погружения с произвольным ядром порядка $p^{n}$.

В более узком случае, когда ядро абелево, из работы вытекает один из известных результатов Дёмушкина-Шафаревича (см. $\left[{ }^{3}\right]$, теорема 3), который здесь получается болеё простым путем. Отметим, что в $\left[{ }^{3}\right]$ второе препятствие не вычисляется, тогда -как в данной работе, благодаря отказу от когомологической техники, строится элемент, от:ечающий за исчезновение второго препятствия.

\section{1. Редукция к задаче погружения с менъшим ядром}

Предположим, что поле $k$ содержит группу корней $p$-й степени из единицы $-\left\{\zeta_{p}\right\}$. Введем в группе характеров группы $N$, т. е. в группе $N^{*}=\operatorname{Hom}\left(N, k^{*}\right)$, операторы из группы $G$ по формуле

$$
\chi^{\sigma}(a)=\left[\chi\left(\sigma a \sigma^{-1}\right)\right]^{\sigma}, \quad \text { где } \chi \in N^{*}, \quad \sigma \in G, \quad a \in N .
$$

Пусть $\chi_{1}$ - центральный характер группы $N$, отличный от единичного, и такой, что $\chi_{1}^{p}=1$, а значит $N_{1}=\operatorname{Ker} \chi_{1}$ имеет в группе $N$ индекс $p$. Такой характер существует, так как характеры группы $N$ образуют $p$-группу. 
Предположжи, что для задачи погружения (1) условие соглазсностй выполнено, и рассмотрим задачу погружения

$$
1 \rightarrow N / N_{1} \rightarrow G / N_{1} \rightarrow \Gamma\left(k / k_{0}\right) \rightarrow 1 .
$$

Решение этой задачи существует и строится следующим об́разом.

Продолжим характер $\chi_{1}$ на алгебру $k N$, полагая $\chi_{1}(x)=x$ для $x \in k$. Введем обозначение $\lambda_{\sigma}=\chi_{1}\left(l_{\sigma}\right)$. Имеем $\lambda_{\sigma} \in k, \quad \lambda_{\sigma_{1}}^{\sigma_{2}} \lambda_{\sigma_{2}}=\lambda_{\sigma_{1} \sigma_{2}}$ и $\left\{\bar{\lambda}_{\tau}\right\}_{\tau \in N}=\left\{\zeta_{p}\right\} . \quad$ Тогда по теореме Шпайзера существует $\mu \in k$ такое, что $\mu^{\sigma-1}=\lambda_{\sigma}^{p} \quad\left(\mu=\sum_{\sigma \in G} \lambda_{\sigma}^{-p}\right)$, и элемент $\mu$ определен однозначно с точностью до множителей из $k_{0}{ }^{*}$. Легко видеть, что поле $k_{1}=k(\theta)$, где $\theta=\sqrt[p]{\mu}, \quad$ с системой автоморфизмов $\theta^{g}=\theta \lambda_{g}$ решает задачу (2).

Таким образом, для решения задачи (1) остается решить задачу погружения

$$
1 \rightarrow N_{1} \rightarrow G \rightarrow F_{1}=\Gamma\left(k_{1} / k_{0}\right) \rightarrow 1
$$

\section{2. Вычисление второго препятствия в задаче погружения с произвольным ядром порядка $p^{n}$}

Выясним, в чем заключается условие согласности для задачи (3). Как и в случае абелева ядра, назовем это условие вторым препятствием для исходной задачи погружения (см. $\left.\left[{ }^{3}\right]\right)$.

Обозначим через $A=G \times k, B=G \times k_{1}$ - скрещенные произведения группы $G$ с полями $k$ и $k_{1}$, а через $Z_{A}, Z_{B}-$ центры алгебр $A$ и $B$.

Второе препятствие состоит в том, что алгебра $B$ должна быть алгеброй матриц порядка $p$ ord $F$ над некоторой своей подалгеброй.

Алгебра $B$ содержит алгебру матриц порядка ord $F$. Этой алгеброй является алгебра $C$, порожденная полем $k$ и элементами $\sigma l_{\sigma} \cdot(C-$ скрещенное произведение поля $k$ со своей группой Галуа при единичных факторах.)

Итак, для вычисления второго препятствия нужно в алгебре $B$ найти подалгебру $D_{B}$, централизатор $C$ в $B$ и условия, при которых она была бы изоморфна матричной алгебре порядка $p$ (см. $\left[{ }^{4}\right]$, теорема A.4.2).

Обозначим через $D_{A}$ централизатор $C$ в $A$. Изучим алгебру $D_{B}$.

1. Ясно, что $D_{A} \subset D_{B}$ и размерность $D_{B}$ в $p$ раз больше размерности $D_{A}$.

Для нахождения $D_{B}$ нам потребуется следующая конструкция: построение элемента, отвечающего за исчезновение второго препятствия.

В алгебре $k N$ рассмотрим автоморфизм $\psi$. $\psi: a \rightarrow a \chi_{1}(a)$, где $a \in N$. Так как $\theta^{a}=a^{-1} \theta a=\theta \lambda_{a}=\theta \chi_{1}\left(a^{-1}\right)$, то автоморфизм $\psi$ равносилен трансформации при помощи элемента $\theta: \theta^{-1} a \theta=a \chi_{1}(a)$.

Введем в рассмотрение элементы $m_{\sigma}=l_{\sigma}{ }^{-1} l_{\sigma}{ }^{4} \lambda_{\sigma}^{-1}$. Имеем $m_{\sigma} \in k N$, $m_{a}=1$ при $a \in N$ и

$$
\begin{aligned}
& m_{\sigma_{1}}^{\sigma_{2} l} m_{\sigma_{2}}=m_{\sigma_{2}}^{-1} l_{\sigma_{1}}^{-1 \sigma_{2}} \underset{\sigma_{1}}{l \psi \sigma_{2}} \lambda_{\sigma_{1}}^{-1 \sigma_{2}} l_{\sigma_{2}}{ }_{\sigma_{2}}^{-1} \underset{\sigma_{2}}{l \psi} \lambda_{\sigma_{3}}^{-1}= \\
& =l_{\sigma_{2}}^{-1} l_{\sigma_{1}}^{-1 \sigma_{2}} \underset{\sigma_{1}}{\Psi \sigma_{2}} l \underset{\sigma_{2}}{\Psi} \lambda_{\sigma_{1} \sigma_{2}}^{-1}=\left(\underset{\sigma_{1} \sigma_{2}}{l \sigma_{2}} l\right)^{-1} \underset{\sigma_{1} \sigma_{2}}{\underset{\psi}{\Psi}} \lambda_{\sigma_{1} \sigma_{2}}^{-1}=m_{\sigma_{1} \sigma_{2}} .
\end{aligned}
$$

Существует $q \in k N$ такое, что $q^{\sigma l} \sigma=m_{\sigma} q$. Действительно, для $q=\sum_{g \in G} m_{g}^{-1}$ это выполнено. Элемент $q$ определен однозначно с точностью 
до правых множителей из $D_{A} \cap k N \supset Z_{A}$. Покажем, что $u=\theta q$ коммутирует со всеми $\sigma l_{\sigma}$.

Действительно,

$$
\theta q \sigma l_{\sigma}=\theta \sigma l_{\sigma} q^{\sigma l} \sigma=\sigma \theta \lambda_{\sigma} l_{\sigma} m_{\sigma} q=\sigma \lambda_{\sigma} l_{\sigma}^{\psi^{-1}} l_{\sigma}^{-1 \psi^{-1}} l_{\sigma} \lambda_{\sigma}^{-1} \theta q=\sigma l_{\sigma} \theta q .
$$

Значит, $D_{B}=D_{A}(u)$, а для всякого $z \in Z_{A} \subset D_{A}$ имеем $u^{-1} z u=z^{\psi}$. Положим $v=u^{p} ; v=q^{1+\psi+\ldots+\psi^{p-1}} \mu$. Нетрудно видеть, что $v \in Z_{B}$, а при изменении $\theta v$ меняется на множитель $c$, пробегающий $k_{0}{ }^{*}$.

2. Обратимся теперь к строению алгебр $Z_{A}, Z_{B}$.

Пусть $X_{j}$ - минимальный левый идеал простой компоненты $(k N)_{j}$ алгебры $k N$. Тогда $(k N)_{j}=(k N) e_{\chi_{j}}$ для некоторого идемпотента $e_{\gamma_{j}}$ центра и, согласно [5] (с. 225),

$$
e_{\chi_{j}}=\left(x_{j} /[N: 1]\right) \sum_{a \in N} a_{\chi_{j}}\left(a^{-1}\right),
$$

где $\chi_{j}$ - характер группы $N$, соответствующий идеалу $X_{j}, x_{j}=\left(X_{j}: k\right)$, причем элементы $e_{\chi_{j}}$ образуют $k$-базис центра $Z_{k N}$ алгебры $k N$.

Обозначим через $M$ множество всех $\chi_{j}$. Так как $Z_{A} \subset Z_{k N}$, то $Z_{A}=\left\{\sum_{\chi \in M} x_{\chi} e_{\chi} \mid, x_{\chi} \in k, \sum x_{\chi}^{g} e_{\chi}^{g}=\Sigma x_{\chi} e_{\chi}\right\}$. Нетрудно проверить, что $e_{\chi}^{g}$ снова является идемпотентом из множества $\left\{e_{\chi}\right\}$. Если положить по определению $\left.\chi^{g}(a)=\chi\left(g^{a g}\right)^{-1}\right)^{g}$ для $a \in N$, то видим, что $e_{\chi^{g}}=e_{\chi^{g}}$. Таким образом, если $\chi \in M$, то $\chi^{g} \in M$.

Итак, $Z_{A}=\left\{\sum_{\chi \in M} x_{\chi} e_{\chi} \mid x_{\chi}^{g}=x_{\chi^{0}}\right\}, \quad$ т. е. $Z_{A}$ распадается в прямую сумму идеалов, натянутых на $e_{\chi}$ и сопряженных так, что прямая сумма таких идеалов, натянутых на идемпотенты, сопряженные с $e_{\chi}$, изоморфна полю $k_{x}$, и каждое такое прямое слагаемое находится в естественном взаимно однозначном соответствии с классами сопряженных характеров. Здесь $k_{\chi}=k^{G} \chi, G_{\chi}=\left\{\sigma \in G \mid \chi^{\sigma}=\chi\right\}, \chi \in M$.

Найдем вид произвольного элемента $z$ из алгебры $Z_{B}$. Легко видеть, что $Z_{B}=\left\{z \in Z_{A} \mid z \theta=\theta z\right\}=\left\{z \in Z_{A}, z=\sum x_{\chi} e_{\chi} \mid x_{\chi}=x_{\chi} \cdot x_{1}^{k}, k=0,1, \ldots\right.$ $\ldots, p-1\}$. Иначе говоря, $Z_{B}$ изоморфна прямой сумме полей $\hat{k}_{\chi}$ и каждое такое прямое слагаемое находится в естественном взаимно однозначном соответствии с классом характеров $\left\{\chi^{\sigma} \chi_{1}^{k}\right\}_{\sigma \in G,}$, т. е. с классом элементов, сопряженных с $\hat{\chi} \in \hat{M}=M /\left\{\chi_{1}\right\}$. Здесь $\hat{k}_{x}=k \hat{G}^{\hat{G}}$, $\hat{G}_{\chi}=\left\{g \in G \mid e_{\chi}^{g}=e_{\chi \chi_{1}^{k}}, k=0,1, \ldots, p-1\right\}$.

3. Имеем $D_{B}=D_{A}(u) \supset Z_{A}(u)$. Будем искать необходимые и достаточные условия того, чтобы алгебра $D_{A}(u)$ была алгеброй матриц порядка $p$.

Есть две возможности.

1) $D_{A}$ - матричная алгебра порядка $p$. Этот случай не интересен, так как приводит к тому, что уже алгебра $A$ становится матричной алгеброй порядка ord $F p$.

2) $D_{A}$ не является матричной алгеброй порядка $p$. Тогда при изоморфизме $D_{A}(u) \approx(\mathfrak{M})_{p} D_{A}$ вкладывается в $\mathfrak{N}$, что влечет за собой изоморфизм

$$
Z_{A}(u) \approx(\Re)_{p}, \quad \text { где } \Re \subset \mathfrak{M} .
$$

Итак, нам необходимо и достаточно найти условия, при которых алгебра $Z_{A}(u)$ становится матричной алгеброй порядка $p$. 
4. Найдем этй условия. Разложим $v$ на компоненты как элемент алгебры $Z_{B} . v=\Sigma v_{\hat{\chi}} e_{\hat{\chi}}$, где $v_{\hat{\chi}} \in \hat{k}_{\chi}, e_{\hat{\chi}}=\sum_{k=0}^{p-1} e_{\chi \chi_{1}^{k}}$.

Как действует на компонентах алгебры $Z_{A}$ автоморфизм $\psi$, индуцированный элементом $\theta$ ? $\mathrm{B}$ соответствии со строением $Z_{A}$ для любого $z \in Z_{A}$ имеем $z=\Sigma x_{\chi} e_{\chi}$, где $x_{\chi} \in k_{\chi}=\hat{k}_{\chi}\left(\sqrt[p]{a_{\hat{\alpha}}}\right)$. Запишем $x_{\chi}$ в виде $x_{\chi}=\sum_{i=0}^{p-1} x_{\chi, i} \sqrt[p]{a_{\hat{\chi}}}{ }^{i}$, где $x_{\chi, i} \in \hat{k}_{\chi}$. Так как $x_{\chi}^{\sigma}=x_{\chi^{\sigma}}$, то при $\sigma \in \hat{G}_{\chi} / G_{\chi}$, т. е. при таком $\sigma$, что $\chi^{\sigma}=\chi^{\psi^{k}}=\chi \cdot \chi_{1}{ }^{k}$, будет выполнено равенство $x_{\chi} \sigma=\Sigma x_{\chi, i}\left(\sqrt[p]{a_{\hat{\chi}}} \zeta_{p}^{k}\right)^{i}, \quad$ где $\zeta_{p}-$ первообразный корень $p$-й степени из 1.

Значит, автоморфизм $\psi$ на компоненте $Z_{A}$, изоморфной полю $\hat{k}_{\chi}\left(\sqrt[p]{a_{\hat{\chi}}}\right)$, производит действие

$$
\sqrt[p]{a_{\hat{\chi}}} \longrightarrow \sqrt[p]{a_{\hat{\chi}}} \zeta_{p}
$$

Поэтому в соответствии с разложением $B$ в прямую сумму простых подалгебр с центрами, являющимися прямыми слагаемыми $Z_{B}$, $Z_{A}(u) \subset B$ разложится в прямую сумму подалгебр, являющихся обобщенными алгебрами кватернионов:

$$
Z_{A}(u) \approx \oplus \Sigma \hat{k}_{\chi}\left[a_{\hat{\chi}}, v_{\hat{\chi}}\right]
$$

Таким образом, для того чтобы при каком-то выборе $\theta$ алгебра $Z_{A}(u)$, а значит и алгебра $D_{B}$, была изоморфна алгебре матриц порядка $p$ над некоторой своей подалгеброй, необходимо и достаточно, чтобы существовало такое $c \in k_{0}$, что

$$
v_{\hat{\chi}} c \in N m \hat{k}_{\chi}\left(\sqrt[p]{a_{\hat{\chi}}}\right) / \hat{k}_{\chi}
$$

причем достаточно брать по одному представителю $\hat{\chi}$ из каждого класса сопряженности $M /\left\{\chi_{1}\right\}$ относительно $G$.

Мы рассматриваем задачу погружения полей, поэтому, если мы хотим получить в качестве решения поле, а не алгебру Галуа, необходимо дополнить полученные условия следующим: $\mu c \notin k^{p}$.

\section{ЛИТЕРАТУРА}

1. Делоне Б. Н., Фаддеев Д. К. Матем. сб., 17, № 1, 243-284 (1944).

2. Hasse, H., Math. Nachr., 1, № 1, 40-61 (1948).

3. Дёмушкин С. П., Шафаревич Н. Р. Изв. АН СССР, сер. матем., 23, № 6, 823-840 (1959).

4. Херстейн Н. Некоммутативные кольца. М., «Мир», 1969.

5. Кэртис Ч., Райнер Н. Теория представлений конечных групп и ассоциативных алгебр. М., «Наука», 1969.

Всесоюзное аэрологическое научно-производственное объединение «Аэрогеология»

Министерства геологии СССР
Поступила в редакцию 29 июня 1981

После переработки 18 мая 1982 


\section{SUVALISE $p^{n}$-JÄRKU TUUMAGA SISESTUSULEASANDESTT}

On käsitletud suvalise $p^{n}$-järku tuumaga $N$ sisestusülesannet, mis on seotud täpse järgnevusega

$$
1 \longrightarrow N \longrightarrow G \stackrel{\varphi}{\longrightarrow} F=\Gamma\left(k / k_{0}\right) \longrightarrow 1
$$

ja mille kohta kehtib kooskõlalisuse tingimus. On vaadeldud elementi $v$ ja leitud, et selle sisestusülesande teise takistuse kadumise jaoks on tarvilik ja piisav sellise $c \in k_{0}$ eksisteerimine, mille puhul

$$
v_{\hat{\chi}} c \in N m k_{\chi} / \hat{k}_{\chi}
$$

kus $v_{\hat{\chi}}$ on elemendi $v$ komponendid ja $k_{\chi}, \hat{k}_{\chi}$ välja $k$ alamväljad.

O. POLEVITSKAYA

\section{ON THE EMBEDDING PROBLEM WITH AN ARBITRARY KERNEL OF ORDER $p^{n}$}

In this paper a field embedding problem associated with an exact sequence

$$
1 \longrightarrow N \longrightarrow G \longrightarrow F=\Gamma\left(k / k_{0}\right) \longrightarrow 1
$$

is considered.

Let $N$ be a group of order $p^{n}$, and it is of no importance whether $N$ is an abelian group or non-abelian. Suppose that $k$ contains a primitive $p$-th root of unity, let char $k$ does not divide ord $N$.

Suppose, also, that a condition of concordance $\left(\left[{ }^{1,2}\right]\right)$ is fulfilled for our problem.

Then the problem (1) can be reduced to a problem with a lesser kernel

$$
1 \longrightarrow N_{1} \longrightarrow G \rightarrow F_{1}=\Gamma\left(k_{1} / k_{0}\right) \rightarrow 1,
$$

where $N_{1}$ is a subgroup of the group $N,\left(N: N_{1}\right)=p, k_{1}=k(\sqrt[p]{\mu c)}, c-$ an arbitrary member of $k_{0}^{*}$.

A condition of concordance for the problem (2) is called a second obstacle for the problem (1).

It is shown in this paper that for disappearance of the second obstacle of the problem (1), it is necessary and sufficient that there is such an element $c \in k_{0}$ that

$$
v_{\hat{x}} c \in N m k_{\chi} / \hat{k}_{\chi}
$$

where $v_{\hat{\chi}}$ are the components of $v$ from some algebra; $k_{\chi}, \hat{k}_{\chi}$ are the subfields of the field $k$. On the basis of non-cohomological wordings of the condition of concordance, $v$ is calculated.

In the more narrow case, when the kernel is abelian, one of the well-known results of Demushkin-Shafarevitch $\left(\left[{ }^{3}\right]\right.$, Theorem 3) can be obtained more simply according to this paper. 